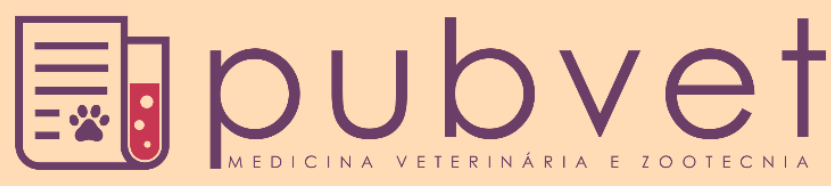

https://doi.org/10.31533/pubvet.v15n07a873.1-8

\title{
Leptospirose caprina: os riscos de uma doença negligenciada
}

\author{
Elisa Cristina Gonçalves Silva ${ }^{1^{*} \bullet}$, Raul José Silva Girio ${ }^{2} \bullet$ (D) \\ ${ }^{1}$ Discente do curso de Medicina Veterinária, Universidade de Marília - Unimar. Marília - SP, Brasil. \\ ${ }^{2}$ Docente do curso de Medicina Veterinária, Universidade de Marília - Unimar. Marília - SP, Brasil. \\ *Autor para correspondência,E-mail: elisa.cgs@hotmail.com
}

Resumo. A leptospirose caracteriza-se como a zoonose bacteriana mais comum, sendo sua perpetuação garantida por condições ambientais favoráveis à sobrevivência das leptospiras, diversidade de sorovares e potenciais hospedeiros existentes. A instalação da enfermidade depende da imunidade inata ou adaptativa e estado fisiológico do hospedeiro, bem como do agente etiológico e características ambientais. Nos caprinos, a doença manifesta-se de forma inaparente, aguda ou crônica de acordo com o tipo de sorovar envolvido e longevidade da infecção. Cada modo de manifestação da leptospirose caprina abrange aspectos clínicos específicos e grande parte dos sinais clínicos detém patogenia desconhecida, pouco elucidada ou baseada em analogia. Disfunções reprodutivas, declínio da produção leiteira, redução na taxa de crescimento e manejo inadequado atuam como fatores limitantes à produção, acarretando prejuízos econômicos.

Palavras-chave: Disfunção reprodutiva, leptospirose caprina, prejuízos econômicos

\section{Goat leptospirosis: the risks of a neglected disease}

Abstract. Leptospirosis is presumed to be the most common bacterial zoonosis. Its perpetuation is assured by environmental conditions favorable to survival of leptospires, great variety of serovars and potential hosts. Illness onset depends on the innate or adaptive immunity and physiological status of the host, as well as causative agent and environmental conditions. In goats, the clinical presentations are either subclinical, acute or chronic depending on serovars involved and duration of disease. Each clinical presentation of goat leptospirosis involves specific clinical aspects and pathogenesis of a large portion of the clinical signs is unknown, not well elucidated or based on analogy. Reproductive dysfunctions, decreased milk production, reduced growth rate and inadequate handling act as limiting factors to production, resulting in economic losses.

Keywords: Reproductive dysfunction, goat leptospirosis, economic losses

\section{Leptospirosis caprina: los riesgos de una enfermedad descuidada}

Resumen. La leptospirosis se caracteriza como una de las zoonosis bacterianas más comunes, que es perpetuada por las condiciones ambientales que favorecen la sobrevivencia de las leptospiras, diversidad de serovares y potenciales hospederos que existen en el medio. La implantación de la enfermedad depende de la inmunidad innata o adaptativa y del estado fisiológico del huésped, bien como del agente etiológico y características ambientales. En los caprinos, esta enfermedad se manifiesta de forma inaparente, aguda o crónica de acuerdo con el tipo de serovar responsable y de la duración de la infección. Cada tipo de manifestación de la leptospira caprina cubre aspectos clínicos específicos y grande parte de los síntomas clínicos mantiene una patogénesis desconocida, poco clara o que se basa en una analogía. Disfunciones reproductivas, la disminución de la producción de leche, la reducción en la tasa de 
crecimiento y el manejo inadecuado actúan como factores limitantes a la producción, trayendo consigo pérdidas económicas.

Palabras clave: Disfunción reproductiva, leptospirosis caprina, pérdidas económicas

\section{Introdução}

A caprinocultura atua nos âmbitos cultural, social e econômico em função do desenvolvimento nordestino (Farias et al., 2014), tendo em vista que o nordeste brasileiro detém acima de $90 \%$ do rebanho nacional e totaliza 10.687.777 caprinos (ANUALPEC, 2020). Existente desde os primórdios da civilização, a relação entre caprinos e o ser humano propicia o suprimento de produtos como leite, carne e pele. Contudo, a atividade no Brasil apresenta fatores limitantes quanto à produtividade como o potencial genético, manejo, gerenciamento reprodutivo, nutrição, qualidade das forrageiras tropicais, clima, sazonalidade da produção e controle das enfermidades (Ribeiro, 1997; Silva et al., 2012).

A leptospirose opera como uma enfermidade de impactos produtivos devido aos neonatos fracos, redução na taxa de crescimento e abortos (Hamond et al., 2014). Como consequências da redução do desempenho reprodutivo estão a queda na produção leiteira e de carne que, por sua vez, geram riscos aos criadores atuantes em países tropicais ou emergentes (Martins et al., 2012b). Nos países em desenvolvimento, especialmente, a caprinocultura possui papel voltado também para a subsistência (Martins \& Lilenbaum, 2014).

\section{Etiologia}

A bactéria que atua como agente etiológico na leptospirose é pertencente à ordem Spirochaetales, família Leptospiraceae e gênero Leptospira (Oliveira et al., 2013). O gênero Leptospira subdivide-se em 22 espécies, mais de 300 sorovares e sorogrupos compostos por sorovares relacionados antigenicamente (Picardeau, 2017) mediante sua estrutura lipopolissacarídica (Picardeau, 2013). Os sorovares existentes enquadram-se em 25 sorogrupos (Rizzo et al., 2017), sendo a compreensão dos sorogrupos e seus reservatórios de grande valia para a epidemiologia da doença em determinada localidade (Martins \& Lilenbaum, 2013).

As espécies podem ser agrupadas em patogênicas e não patogênicas de acordo com a semelhança de seu material genético (Martins \& Lilenbaum, 2014). Por intermédio de técnicas moleculares, houve uma reclassificação do gênero Leptospira que resultou em 19 genomespécies (Higino \& Azevedo, 2014). As espécies patogênicas são representadas por Leptospira interrogans, Leptospira kirschneri, Leptospira noguchii, Leptospira alexanderi, Leptospira weilii, Leptospira borgpetersenii, Leptospira santarosai, Leptospira kmetyi, Leptospira canicola, Leptospira grippotyphosa, Leptospira icterohaemorrhagiae e Leptospira pomona. As não patogênicas, por sua vez, são compostas por Leptospira biflexa, Leptospira meyeri e Leptospira wolbachii (Genovez, 2016). Ainda, existem as espécies intermediárias, cuja patogenicidade é pouco elucidada e o grupo é compreendido por Leptospira inadai, Leptospira broomii, Leptospira fainei, Leptospira wolffii e Leptospira licerasiae (Bourhy et al., 2013).

Em 2007, foram isoladas duas amostras de caprinos brasileiros contendo Leptospira spp. e as mesmas são tidas como os primeiros isolados de origem caprina em território nacional. As amostras foram submetidas a testes moleculares e o resultado originou um novo tipo denominado Carioca (Lilenbaum et al., 2014). No Brasil, os sorovares mais frequentes em rebanhos caprinos são Australis Bratislava, Australis Australis, Autumnalis Autumnalis, Autumnalis Butembo, Ballum Castelonnis, Bataviae Bataviae, Canicola Canicola, Grippotyphosa Grippotyphosa, Icterohaemorrhagiae Icterohaemorrhagiae, Panama Panama, Pomona Pomona, Pyrogenes Pyrogenes, Sejroe Hardjo, Sejroe Wolfii e Tarassovi Tarassovi (Santos et al., 2012). Em especial, leptospiras identificadas como Sejroe Hardjo são comumente associadas à leptospirose caprina e os pequenos ruminantes atuam como potenciais reservatórios do sorovar Hardjo (Martins \& Lilenbaum, 2014), ou seja, o sorovar evidenciado é adaptado ao hospedeiro (Cortizo et al., 2015). Vale salientar que o patógeno pode ser classificado fenotipicamente em sorogrupos/sorovares e genotipicamente em genótipos. Embora a associação de ambas as ferramentas seja complementar (Jaeger et al., 2019), suas conclusões são frequentemente divergentes (Lilenbaum et al., 2014). 
Como características de tais bactérias, é possível citar a morfologia espiral fina, dimensão de 6 a 20 $\mu \mathrm{m} \times 0,1 \mu \mathrm{m}$, presença de cilindro citoplasmático, bainha externa, gancho em suas extremidades, endoflagelos, alta mobilidade, classificação gram-negativa, aerobiose obrigatória, oxidase e catalase positivas. Ressalta-se, ainda, que alguns sorovares do gênero podem apresentar hemolisina esfingomielinase $\mathrm{C}$ em sua parede celular, atividade lipase e produção de urease (Quinn et al., 2005). Picardeau (2017) também afirma que o alongamento celular se dá em razão da síntese de peptideoglucano de forma dispersa ou lateral e, em relação à adaptação da bactéria em diversos hospedeiros, sugere-se que ocorra devido à transferência horizontal de genes, duplicação de genes e redução do genoma.

As condições favoráveis para a sobrevivência das leptospiras incluem solo alcalino ou neutro, solo de origem vulcânica (Schneider et al., 2013), umidade elevada, temperatura próxima aos $20^{\circ} \mathrm{C}$ e proteção contra radiação solar. Esses fatores, juntamente à ampla gama de sorovares e potenciais hospedeiros, garantem a perpetuação da doença (Higino \& Azevedo, 2014) apesar da incapacidade da bactéria de perdurar em altas densidades no solo ou água (Gostic et al., 2019), água salgada (Grennan, 2019), pH ácido, sob radiação solar direta e em ambientes secos (Genovez, 2016).

\section{Patogenia e aspectos clínicos}

A leptospirose ilustra o conceito de saúde única, visto que na mesma há interação entre animais, seres humanos e ecossistemas (Schneider et al., 2013). Sua soroprevalência em determinada área depende, basicamente, de três elementos: agente etiológico, características ambientais e suscetibilidade do hospedeiro (Carvalho et al., 2014). Ademais, as bactérias mantidas pelo hospedeiro são nocivas quando esse se encontra imunocomprometido, atingindo fêmeas no curso final da gestação, neonatos e animais que apresentam infecções concomitantes (Ellis, 2015).

De caráter zoonótico, a leptospirose animal difere da humana quanto à patogenia, sinais clínicos, requisitos dos métodos diagnósticos, medidas de controle e aspectos epidemiológicos. Relatos de leptospirose animal existem na maior parte do globo (exceto nas regiões polares) e em praticamente todas as espécies animais investigadas (Ellis, 2015), caracterizando-a como a zoonose bacteriana mais comum. A infecção é capaz de acometer mamíferos, pássaros, anfíbios, répteis e, possivelmente, peixes (Picardeau, 2017).

Nos caprinos, a apresentação da doença se dá de forma inaparente, aguda ou crônica. A manifestação inaparente é a mais recorrente e responsável pela manutenção do agente etiológico no rebanho (Higino \& Azevedo, 2014), sendo suas consequências sobre o gado subestimadas. As leptospiras adaptadas aos hospedeiros que as transmitem costumam ocasionar infecção subclínica e constituem uma fonte de infecção tanto para animais quanto para os humanos. Já a forma aguda é desencadeada por infecções incidentais envolvendo leptospiras transportadas pelos demais animais domésticos ou de vida livre atuantes como reservatórios e resulta em surtos com frequência (Martins \& Lilenbaum, 2014). Em ruminantes, as infecções incidentais são atípicas, enquanto a cronicidade é configurada por infecções envolvendo cepas adaptadas que promovem sinais clínicos de grau leve e disfunções reprodutivas (Martins \& Lilenbaum, 2013).

O sorovar Hardjo é integrante do sorogrupo Sejroe, adaptado aos caprinos e endêmico na espécie, sendo comumente associado aos animais subclínicos apresentando transtorno reprodutivo (Cortizo et al., 2015). Cepas adaptadas como as integrantes do sorogrupo Sejroe são predominantes no meio rural, ao passo em que centros urbanos são afetados, majoritariamente, por Icterohaemorrhagiae (Martins \& Lilenbaum, 2013). De acordo com observações envolvendo animais e seres humanos, é possível afirmar que cepas intermediárias desencadeiam manifestações clínicas leves nos mesmos (Picardeau, 2017).

A transmissão da leptospirose ocorre através do contato com fontes contaminadas tais como solo, água, urina (Costa et al., 2016), produtos da procriação e alimentos (Phillips, 2019), assim como as transmissões sexual e vertical são passíveis de verificação (Ellis, 2015). Inseminação artificial e transferência de embrião tornam-se técnicas capazes de transmitir a bactéria caso antibióticos não sejam utilizados no processo (Loureiro \& Lilenbaum, 2020). Por outro lado, o ser humano raramente é transmissor da doença devido ao seu status de hospedeiro incidental na maioria dos casos (Hamond et 
al., 2014). Para o estabelecimento da infecção, entretanto, é necessário que o agente etiológico realize a travessia de barreiras físicas como pele e mucosas, as quais constituem a principal linha de defesa imunológica eficiente. Assim, a perda da integridade de barreiras físicas favorece a instalação da infecção e, quando comparada à pele ilesa, requer uma quantidade inferior de antígeno para infectar o animal (Gostic et al., 2019).

Ainda de acordo com Gostic et al. (2019), após transpor as barreiras físicas, o adentramento no organismo do hospedeiro expõe o agente etiológico às ações da imunidade inata ou adaptativa e sua sobrevivência aos ataques imunes consiste num passo fundamental para que a infecção se inicie. Na ausência de anticorpos específicos, as leptospiras patogênicas persistem à fagocitose de macrófagos e neutrófilos, podendo também sobreviver no interior de fagócitos e evadir-se posteriormente através da indução do processo de apoptose (Adler, 2014). Outro feito das bactérias é a sobrevivência diante do sistema complemento com subsequente disseminação sanguínea (Fernandes et al., 2016) e linfática, resultando no acometimento de órgãos como pulmões, fígado e baço (Genovez, 2016).

A bacteremia ocorre durante a fase aguda da leptospirose, na qual prevalece a infecção de animais jovens e, geralmente, estão envolvidas cepas incidentais produtoras de hemolisinas. Nesse estágio, é possível verificar a presença do agente etiológico tanto no sangue quanto no leite, a leptospiremia materna enquadra-se como a responsável pela infecção transplacentária (Ellis, 2015) e a progressão resulta na leptospirose crônica com disfunções reprodutivas aparentes (Loureiro \& Lilenbaum, 2020), bem como leptospirúria intermitente nos animais sobreviventes (Oliveira et al., 2013).

O estágio de leptospiremia ocorre de sete a dez dias após a infecção (Martins \& Lilenbaum, 2014) e tem duração aproximada de quatro a cinco dias, caracterizando-se por multiplicação bacteriana na corrente sanguínea e em diversos órgãos. As leptospiras, então, deslocam-se para a luz dos túbulos renais, aparelho reprodutor, câmara anterior do globo ocular e sistema nervoso central ao passo em que o hospedeiro desenvolve uma resposta imunológica, já que em tais locais a imunidade humoral é pouco ou não atuante. A leptospirúria, por sua vez, é caracterizada pela localização renal, início entre o sétimo e o décimo dia de evolução da doença, presença de complexos imunes e inflamação com consequente vasculite generalizada. Rins, sistema reprodutor, fígado, coração e pulmões são os principais órgãos acometidos pela vasculite (Higino \& Azevedo, 2014).

A persistência renal da bactéria ocorre pois, embora a urina contenha anticorpos sorovarespecíficos contra LPS, a ausência de fagócitos possibilita a multiplicação e excreção bacterianas. Quanto ao status de portador renal e/ou genital, o caráter assintomático é garantido devido ao fato de que as leptospiras podem se encontrar recobertas por proteínas ou polissacarídeos do hospedeiro e, dessa forma, tornam-se não aglutináveis ao anticorpo específico (Genovez, 2016). Ademais, Ellis (2015) relata que tanto a duração quanto a intensidade da leptospirúria apresentam variações de acordo com a individualidade animal e o sorovar infectante.

No âmbito clínico, anorexia, dificuldade respiratória, febre, anemia hemolítica, icterícia (Higino et al., 2012), depressão, síndromes hemorrágicas (Costa et al., 2016), irritabilidade, diarreia, opacidade na pele, síndrome da queda do leite, abortos epidêmicos (Martins \& Lilenbaum, 2014), nascimento de crias fracas ou natimortos, infertilidade (Araújo Neto et al., 2010), presença de sangue na bexiga, hepatomegalia, hemoglobinúria, rins aumentados, miocardite e meningite são sinais compatíveis com a fase aguda da leptospirose caprina. Por meio de cortes histológicos, é possível observar lesão das células endoteliais de pequenos vasos sanguíneos, dissociação hepatocelular, colestase intracanicular, necrose hepatocelular, hipertrofia das células de Kupffer, nefrite intersticial aguda, degeneração tubular/glomerular e infiltrado linfoplasmocitário, assim como infiltrado de macrófagos (Ellis, 2015).

A leptospirose caprina crônica é predominante quando o agente etiológico corresponde a um sorovar adaptado à espécie. Nota-se as seguintes alterações patológicas: abortos espontâneos, natimortos, elevada taxa de mortalidade durante os primeiros dias de vida (Costa et al., 2016), nascimento de cria fraca ou prematura, mumificação fetal (Martins \& Lilenbaum, 2013), repetição do estro, retenção placentária (Campos et al., 2017), reabsorção embrionária, maceração fetal (Martins et al., 2012a), redução da produção leiteira, agalactia (Higino \& Azevedo, 2014), intervalos de parto prolongados, aumento do número de serviços por concepção, infertilidade (Martins \& Lilenbaum, 2014), doença neonatal e lesões renais representadas por pequenos focos cinzentos associados ou não 
a um anel de hiperemia circundante. A histologia aponta nefrite intersticial focal progressiva com infiltração leucocitária, sendo lesões mais antigas caracterizadas por fibrose e infiltrado intersticial (Ellis, 2015). Além disso, Higino \& Azevedo (2014) destacam a presença de atrofia glomerular, manguitos perivasculares e hemorragia cerebral.

A patogênese dos sinais reprodutivos previamente expostos e a localização precisa da infecção genital da fêmea não estão totalmente esclarecidas, porém sugere-se que as bactérias situadas no útero e trato genital interfiram no implante e na gestação (Martins \& Lilenbaum, 2014), além de atingirem células embrionárias através de penetração da zona pelúcida (Ellis, 2015).

Vale frisar que, embora a leptospirose apresente riscos aos produtores, informações a respeito da doença em caprinos são limitadas e baseiam-se, principalmente, em estudos utilizando bovinos (Martins \& Lilenbaum, 2014). Por outro lado, há indicativos de que a espécie detém menor suscetibilidade quando comparada às demais (Santos et al., 2012).

\section{Fatores de risco}

Como fatores de risco para o desenvolvimento da leptospirose, os caprinos apresentam a idade adulta, raça definida, mão de obra contratada, criação consorciada, temperaturas elevadas, alta umidade, diversidade de sorovares existentes na região, presença de roedores, instalações precárias, manejo inadequado, sistema de produção intensivo (Santos et al., 2012) ou semi-intensivo (Cortizo et al., 2015), elevada pluviosidade (Costa et al., 2016), dieta a base de concentrado, contêiner compartilhado (Topazio et al., 2015), pastejo ultrapassando duas horas diárias (Higino \& Azevedo, 2014), acesso a pastagens e assistência veterinária ausente ou infrequente (Rizzo et al., 2017). Araújo Neto et al. (2010) sugerem como fatores de risco relacionados à espécie a alocação em propriedades que contêm cães e bovinos, assim como o contato com animais selvagens e roedores.

\section{Métodos de controle e profilaxia}

Programas apropriados visando reduzir a incidência de leptospirose nos pequenos ruminantes situados em regiões tropicais são obrigatórios quando o objetivo é aumentar a produtividade, seja essa de leite ou carne (Martins et al., 2012b). Nesse sentido, adota-se a desratização e antirratização, higienização das instalações, isolamento dos animais portadores e doentes, drenagem de pastos, realização de exames previamente à introdução de animal no rebanho, introdução de novos animais no rebanho seis meses após a ocorrência de surto, estabelecimento de normas sanitárias em processos de inseminação artificial, fornecimento tanto de água quanto de alimentos de qualidade e separação associada à monitorização de fêmeas prenhes (Genovez, 2016) como medidas a serem continuadas (Martins et al., 2012a). São também implementados o pastejo rotacionado (Babylon et al., 2018), saneamento ambiental, eliminação de pilhas de material descartado, contenção de pragas, proibição de consorciação, manutenção de rebanhos fechados, restrição do acesso animal a fontes de água contaminada, fornecimento de suplemento vitamínico/mineral, quimioprofilaxia (Pimenta et al., 2019), tratamento das fontes de infecção, quarentena voltada aos animais recém adquiridos e vacinação envolvendo os sorovares presentes no rebanho (Martins \& Lilenbaum, 2014). Entre as medidas de controle voltadas aos caprinos, a sorologia é crucial (Rizzo et al., 2017) para a determinação de estratégias adequadas ao relacionar o sorovar e seu respectivo reservatório (Santos et al., 2012) com o ambiente em condições tropicais (Martins \& Lilenbaum, 2013).

$\mathrm{Na}$ leptospirose aguda, o tratamento se resume em antibioticoterapia, tratamento suporte e vacinação. Os antibióticos administrados são penicilina associada à estreptomicina, ampicilina, amoxicilina, tetraciclinas, tulatromicina e cefalosporinas de terceira geração. Como tratamento suporte, enquadram-se a transfusão sanguínea, fluidoterapia e diálise. A vacinação, juntamente à antibioticoterapia, evita a cronificação com sua afecção reprodutiva característica e deve ser destinada a todos os animais de risco (Ellis, 2015). Genovez (2016), em contrapartida, preconiza como antibioticoterapia voltada à fase aguda a administração via intramuscular de di-hidroestreptomicina na dose diária de $25 \mathrm{mg} / \mathrm{kg}$ durante três a cinco dias consecutivos, objetivando-se extinguir os sinais clínicos, estado de portador e risco de transmissão aos humanos. 
Quanto à leptospirose crônica, Ellis (2015) aponta como tratamento a utilização de antibióticos e vacinação dos animais de risco. A antibioticoterapia é voltada aos casos de afecções renal e genital crônicas, sendo os portadores renais amplamente tratados com $25 \mathrm{mg} / \mathrm{kg}$ de estreptomicina. Nessa fase, a terapia visa reduzir a eliminação crônica dos portadores e consequente contaminação ambiental (Murphy, 2018). Além disso, o emprego de penicilina associada à estreptomicina no diluente de sêmen se mostra efetivo na destruição de leptospiras (Ellis, 2015).

A imunização é instituída de forma sistemática com o emprego de vacinas comerciais, as quais devem abranger os sorovares circulantes no rebanho (Martins \& Lilenbaum, 2014). Em seu estudo, Martins et al. (2012a) empregaram reforço vacinal 60 dias após a primeira imunização. Associada ao esquema vacinal, a antibioticoterapia preconizada pelos autores foi a administração de $25 \mathrm{mg} / \mathrm{kg}$ de dihidroestreptomicina em dose única. Vale salientar que as vacinas existentes preparadas a partir de células ou membranas inativadas de leptospiras patogênicas não induzem proteção a longo prazo nem conferem imunização cruzada contra sorovares não inclusos na preparação vacinal (Vieira et al., 2014).

\section{Considerações finais}

A caprinocultura destaca-se como uma atividade rentável na extensão de todo o território nacional, principalmente no nordeste brasileiro. Nesse cenário, existem aspectos desfavoráveis à lucratividade produtiva como a negligenciada leptospirose. Contudo, embora apresente interferências tanto reprodutivas quanto produtivas, informações específicas a respeito da afecção de caprinos são escassas e os conteúdos disponíveis, por muitas vezes, baseiam-se em analogia. Dado o exposto, é pertinente enfatizar a importância da profilaxia e realização de testes sorológicos no rebanho.

\section{Referências}

Adler, B. (2014). Pathogenesis of leptospirosis: cellular and molecular aspects. Veterinary Microbiology, 172(3-4), 353-358. DOI: https://doi.org/10.1016/j.vetmic.2014.06.015

ANUALPEC. (2020). Anuário da Pecuária Brasileira (20th ed., Vol. 1). Instituto FNP.

Araújo Neto, J. O., Alves, C. J., Azevedo, S. S., Silva, M. L. C. R., \& Batista, C. S. A. (2010). Soroprevalência da leptospirose em caprinos da microrregião do Seridó Oriental, Estado do Rio Grande do Norte, Brasil, e pesquisa de fatores de risco. Brazilian Journal of Veterinary Research and Animal Science, 47(2), 144-148. DOI: https://doi.org/10.11606/issn.16784456.bjvras.2010.26839

Babylon, A. M., Roberts, M. G., \& Wake, G. C. (2018). Modelling leptospirosis in livestock. Theoretical Population Biology, 121, 26-32. DOI: https://doi.org/10.1016/j.tpb.2018.03.005

Bourhy, P., Vray, M., \& Picardeau, M. (2013). Evaluation of an in-house ELISA using the intermediate species Leptospira fainei for diagnosis of leptospirosis. Journal of Medical Microbiology, 62(6), 822-827. https://doi.org/10.1099/jmm.0.054304-0

Campos, Â. P., Miranda, D. F. H., Rodrigues, H. W. S., Lustosa, M. da S. C., Martins, G. H. C., Mineiro, A. L. B. B., Castro, V., Azevedo, S. S., \& Silva, S. M. M. S. (2017). Seroprevalence and risk factors for leptospirosis in cattle, sheep, and goats at consorted rearing from the State of Piauí, northeastern Brazil. Tropical Animal Health and Production, 49(5), 899-907. DOI: https://doi.org/10.1007/s11250-017-1255-2

Carvalho, S. M., Mineiro, A. L. B. B., Castro, V., Genovez, M. E., Azevedo, S. S., \& Costa, F. A. L. (2014). Leptospirosis seroprevalence and risk factors for sheep in Maranhão state, Brazil. Tropical Animal Health and Production, 46(2), 491-494. DOI: https://doi.org/10.1007/s11250-013-0505-1

Cortizo, P., Loureiro, A. P., Martins, G., Rodrigues, P. R., Faria, B. P., Lilenbaum, W., \& Deminicis, B. B. (2015). Risk factors to incidental leptospirosis and its role on the reproduction of ewes and goats of Espírito Santo state, Brazil. Tropical Animal Health and Production, 47(1), 231-235. DOI: https://doi.org/10.1007/s11250-014-0684-4

Costa, D. F., Silva, A. F., Farias, A. E. M., Brasil, A. W. L., Santos, F. A., Guilherme, R. F., Azevedo, S. S. \& Alves, C. J. (2016). Serological study of the Leptospira spp. infection in sheep and goats 
slaughtered in the State of Paraíba, semiarid of Northeastern Brazil. Semina: Ciências Agrárias, 37, 819-828. DOI: http://dx.doi.org/10.5433/1679-0359.2016v37n2p819.

Ellis, W. A. (2015). Animal leptospirosis. Leptospira and Leptospirosis, 387, 99-137. DOI: https://doi.org/10.1007/978-3-662-45059-8 6

Farias, J. L. S., Araújo, M. R. A., Lima, A. R., Alves, F. S. F., Oliveira, L. S., \& Souza, H. A. (2014). Análise socioeconômica de produtores familiares de caprinos e ovinos no semiárido cearense, Brasil. Archivos de Zootecnia, 63(241), 13-24. DOI: https://doi.org/10.21071/az.v63i241.559

Fernandes, L. G., Siqueira, G. H., Teixeira, A. R. F., Silva, L. P., Figueredo, J. M., Cosate, M. R., Vieira, M. L., \& Nascimento, A. L. T. O. (2016). Leptospira spp.: Novel insights into hostpathogen interactions. Veterinary Immunology and Immunopathology, 176, 50-57. DOI: https://doi.org/10.1016/j.vetimm.2015.12.004

Genovez, M. E. (2016). Leptospirose em animais de produção. In J. Megid, M. G. Ribeiro, \& A. C. Paes (Eds.), Doenças Infecciosas em Animais de Produção e de Companhia (pp. 378-386). Roca, Brasil.

Gostic, K. M., Wunder Junior, E. A., Bisht, V., Hamond, C., Julian, T. R., Ko, A. I., \& Lloyd-Smith, J. O. (2019). Mechanistic dose-response modelling of animal challenge data shows that intact skin is a crucial barrier to leptospiral infection. Philosophical Transactions of the Royal Society B, 374(1782), 20190367. DOI: https://doi.org/10.1098/rstb.2019.0367

Grennan, D. (2019). Leptospirosis. Journal of the American Medical Association, 321(812). DOI: 10.1001/jama.2019.0697

Hamond, C., Martins, G., Loureiro, A. P., Pestana, C., Lawson-Ferreira, R., Medeiros, M. A., \& Lilenbaum, W. (2014). Urinary PCR as an increasingly useful tool for an accurate diagnosis of leptospirosis in livestock. Veterinary Research Communications, 38(1), 81-85. DOI: https://doi.org/10.1007/s11259-013-9582-x

Higino, Severino S S, Alves, C. J., Santos, C. S. A. B., Vasconcellos, S. A., Silva, M. L. C. R., Brasil, A. W. L., Pimenta, C. L. R. M., \& Azevedo, S. S. (2012). Prevalência de leptospirose em caprinos leiteiros do semiárido paraibano. Pesquisa Veterinária Brasileira, 32(3), 199-203. DOI: https://doi.org/10.1590/s0100-736x2012000300003

Higino, Severino Silvano Santos, \& Azevedo, S. S. (2014). Leptospirosis in small ruminants: current epidemiological situation in Brazil. Arquivos DOI: https://doi.org/10.1590/S180816572014000100017

Jaeger, L. H., Pestana, C. P., Correia, L. F. L., Carvalho-Costa, F. A., Medeiros, M. A., \& Lilenbaum, W. (2019). Novel MLST sequence types of pathogenic Leptospira spp.: Opening the black box of animal leptospirosis in Brazil. Acta Tropica, 196, 135-141. DOI: https://doi.org/10.1016/j.actatropica.2019.05.025

Lilenbaum, W., Kremer, F., Ristow, P., Dellagostin, O., Bourhy, P., Hartskeerl, R., \& Vasconcellos, S. (2014). Molecular characterization of the first leptospires isolated from goats in Brazil. Brazilian Journal of Microbiology, 45(4), 1527-1530. DOI: https://doi.org/10.1590/s151783822014000400050

Loureiro, A. P., \& Lilenbaum, W. (2020). Genital bovine leptospirosis: A new look for an old disease. Theriogenology, 141, 41-47.DOI: https://doi.org/10.1016/j.theriogenology.2019.09.011

Martins, G, Brandão, F. Z., Hamond, C., Medeiros, M., \& Lilenbaum, W. (2012a). Diagnosis and control of an outbreak of leptospirosis in goats with reproductive failure. The Veterinary Journal, 193(2), 600-601. DOI: https://doi.org/10.1016/j.tvj1.2012.01.016

Martins, Gabriel, \& Lilenbaum, W. (2013). The panorama of animal leptospirosis in Rio de Janeiro, Brazil, regarding the seroepidemiology of the infection in tropical regions. BMC Veterinary Research, 9(1), 1-7. DOI: https://doi.org/10.1186/1746-6148-9-237

Martins, G. \& Lilenbaum, W. (2014). Leptospirosis in sheep and goats under tropical conditions. Tropical Animal Health and Production, 46(1), 11-17. DOI: https://doi.org/10.1007/s11250-013$\underline{0480-6}$

Martins, G., Penna, B., Hamond, C., Leite, R. C.-K., Silva, A., Ferreira, A., Brandão, F., Oliveira, F., 
\& Lilenbaum, W. (2012b). Leptospirosis as the most frequent infectious disease impairing productivity in small ruminants in Rio de Janeiro, Brazil. Tropical Animal Health and Production, 44(4), 773-777. DOI: https://doi.org/10.1007/s11250-011-9964-4

Murphy, K. (2018). Dealing with leptospirosis in dogs. The Veterinary Record, 183(12), 384-385. DOI: https://doi.org/10.1136/vr.k4093

Oliveira, S. V., Arsky, M. L. N. S., \& Caldas, E. P. (2013). Reservatórios animais da leptospirose: Uma revisão bibliográfica. Saúde (Santa Maria), 39(1), 9-20. DOI: https://doi.org/10.5902/223658345094

Phillips, J. A. (2019). Leptospirosis. Workplace Health and Safety, 67, 148-148. DOI: https://doi.org/10.1177/2165079918818582

Picardeau, M. (2013). Diagnosis and epidemiology of leptospirosis. Médecine et Maladies Infectieuses, 43(1), 1-9. DOI: https://doi.org/10.1016/j.medmal.2012.11.005

Picardeau, M. (2017). Virulence of the zoonotic agent of leptospirosis: still terra incognita? Nature Reviews Microbiology, 15(5), 297-307. DOI: https://doi.org/10.1038/nrmicro.2017.5

Pimenta, C. L. R. M., Costa, D. F., Silva, M. L. C. R., Pereira, H. D., Júnior, J. P. A., Malossi, C. D., Ullmann, L. S., Alves, C. J., \& Azevedo, S. S. (2019). Strategies of the control of an outbreak of leptospiral infection in dairy cattle in Northeastern Brazil. Tropical Animal Health and Production, 51(1), 237-241. DOI: https://doi.org/10.1007/s11250-018-1635-2

Quinn, P. J., Markey, B. K., Carter, M. E., Donnelly, W. J., \& Leonard, F. C. (2005). Microbiologia veterinária e doenças infecciosas. Artmed.

Ribeiro, S. D. A. (1997). Caprinocultura: Criação Racional de Caprinos. Nobel.

Rizzo, H., Silva, T. R., Carvalho, J. S., Marinho, F. A., Santos, H. A., Silva Júnior, W. S., Alemán, M. A. R., Pinheiro Júnior, J. W., \& Castro, V. (2017). Frequency of and risk factors associated to Leptospira spp. seropositivity in goats in the state of Sergipe, Northeastern Brazil. Ciência Rural, 47(7). DOI: https://doi.org/10.1590/0103-8478cr20160845

Santos, J. P., Lima-Ribeiro, A. M. C., Oliveira, P. R., Santos, M. P., Ferreira Júnior, Á., Medeiros, A. A., \& Tavares, T. C. F. (2012). Seroprevalence and risk factors for leptospirosis in goats in Uberlândia, Minas Gerais, Brazil. Tropical Animal Health and Production, 44(1), 101-106. DOI: https://doi.org/10.1007/s11250-011-9894-1

Schneider, M. C., Jancloes, M., Buss, D. F., Aldighieri, S., Bertherat, E., Najera, P., Galan, D. I., Durski, K., \& Espinal, M. A. (2013). Leptospirosis: a silent epidemic disease. Nternational Journal of Environmental Research and Public Health, 10, 7229-7234. DOI: https://doi.org/10.3390/ijerph10127229

Silva, H. W., Guimarães, C. R. B., \& Oliveira, T. S. (2012). Aspectos da exploração da caprinocultura leiteira no Brasil. Revista Brasileira de Agropecuária Sustentável, 2, 121-125. DOI: https://doi.org/10.21206/rbas.v2i2.173

Topazio, J., Tonin, A. A., Machado, G., Noll, J. C. G., Ribeiro, A., Moura, A. B., Carmo, G. M., Grosskopf, H. M., Martins, J. L. R., \& Badke, M. R. T. (2015). Antibodies to Leptospira interrogans in goats and risk factors of the disease in Santa Catarina (West side), Brazil. Research in Veterinary Science, 99, 53-57. DOI: https://doi.org/10.1016/j.rvsc.2015.01.014

Vieira, M. L., Fernandes, L. G., Domingos, R. F., Oliveira, R., Siqueira, G. H., Souza, N. M., Teixeira, A. R. F., Atzingen, M. V, \& Nascimento, A. L. T. O. (2014). Leptospiral extracellular matrix adhesins as mediators of pathogen-host interactions. FEMS Microbiology Letters, 352(2), 129139. DOI: https://doi.org/10.1111/1574-6968.12349

Histórico do artigo:

Recebido: 11 de fevereiro de2021.

Aprovado: 22 de maio março de 2021.
Licenciamento: Este artigo é publicado na modalidade Acesso Aberto sob a licença Creative Commons Atribuição 4.0 (CC-BY 4.0), a qual permite uso irrestrito, distribuição, reprodução em qualquer meio, desde que o autor e a fonte sejam devidamente creditados. 